\title{
Factores que diferencian el consumo frecuente $y$ esporádico de maribuana en estudiantes universitarios
}

\author{
Angelina Pilatti; Gabriela Rivarola Montejano; Macarena Nillus; Macarena Fernandez; Ricardo Marcos Pautassi \\ Cómo citar este artículo: \\ Pilatti, A., Rivarola Montejano, G., Nillus, M., Fernandez, M., \& Marcos Pautassi, R. M. (2022). Factores que diferencian \\ el consumo frecuente y esporádico de marihuana en estudiantes universitarios. Acta Colombiana de Psicología, 25(1), \\ 87-104. https://www.doi.org/10.14718/ACP.2022.25.1.7
}

Recibido, diciembre 23/2020; Concepto de evaluación, julio 19/2021; Aceptado, septiembre 13/2021

\author{
Angelina Pilatti ${ }^{1}$ \\ ORCID: https://orcid.org/0000-0002-7277-0835 \\ Universidad Nacional de Córdoba, Córdoba, Argentina. \\ Instituto de Investigaciones Psicológicas, IIPsi-CONICET-UNC, Argentina. \\ Gabriela Rivarola Montejano \\ ORCID: https://orcid.org/0000-0003-3323-7293 \\ Universidad Nacional de Córdoba, Córdoba, Argentina. \\ Instituto de Investigaciones Psicológicas, IIPsi-CONICET-UNC, Argentina. \\ Macarena Nillus \\ ORCID: https://orcid.org/0000-0002-8562-1564 \\ Universidad Nacional de Córdoba, Córdoba, Argentina. \\ Instituto de Investigaciones Psicológicas, IIPsi-CONICET-UNC, Argentina. \\ Macarena Fernandez \\ ORCID: https://orcid.org/0000-0001-6179-0921 \\ Universidad Nacional de Córdoba, Córdoba, Argentina. \\ Instituto de Investigaciones Psicológicas, IIPsi-CONICET-UNC, Argentina. \\ Ricardo Marcos Pautassi \\ ORCID: https://orcid.org/0000-0003-1876-2321 \\ Universidad Nacional de Córdoba, Córdoba, Argentina. \\ Instituto de Investigación Médica M. y M. Ferreyra, INIMEC-CONICET-UNC, Córdoba, Argentina..
}

\section{Resumen}

\begin{abstract}
Aunque el consumo de marihuana es altamente prevalente entre universitarios de Argentina, poco se sabe respecto a los factores que permiten distinguir a los consumidores frecuentes de marihuana de quienes consumen esporádicamente. Por esta razón, en el presente trabajo se indagó sobre las posibles variaciones en los contextos de consumo, motivos de consumo, normas percibidas y estrategias conductuales de protección entre universitarios con consumo frecuente o esporádico de marihuana, y se analizó la utilidad de este conjunto de variables para distinguir entre estos los tipos de consumidores mencionados. Para ello, se contó con una muestra de 1083 estudiantes universitarios argentinos que completó una encuesta en línea sobre el consumo de sustancias. En particular, este trabajo se realizó con la submuestra que reportó consumir marihuana en los últimos 30 días ( $n=158 ; 51.3 \%$ mujeres). Dicha encuesta interrogaba sobre el consumo de marihuana y las consecuencias negativas asociadas, así como por motivos de consumo, normas sociales percibidas y estrategias conductuales de protección. Como resultado, los consumidores frecuentes presentaron mayor cantidad de consecuencias negativas $(M=4.92)$ que los consumidores esporádicos $(M=1.82 ; p \leq .05)$, y se halló un perfil distintivo en un conjunto de variables. A nivel multivariado, los motivos de animación ( $\mathrm{OR}=1.230 ; p \leq .05)$, las estrategias conductuales de protección $(\mathrm{OR}=.941 ; p \leq .05)$, el consumo en solitario $(\mathrm{OR}=2.024 ; p \leq .05)$ y el consumo en fiestas universitarias $(\mathrm{OR}=2.669 ; p \leq .05)$ discriminaron significativamente entre ambas clases de consumidores. En general, los hallazgos revelaron subpoblaciones heterogéneas de consumidores de marihuana que difieren no solo en el consumo y sus consecuencias, sino también en los contextos de consumo y en un conjunto de variables relevantes. Estas subpoblaciones distintivas requieren de intervenciones diferentes y específicas.

Palabras clave: factores de riesgo, factores protectores, marihuana, estudiantes.
\end{abstract}

\footnotetext{
${ }^{1}$ Instituto de Investigaciones Psicológicas, IIPSI, Unidad Ejecutora CONICET. Córdoba, Argentina. Facultad de Psicología, Universidad Nacional de Córdoba. Boulevard de la Reforma y Enfermera Gordillo s/n, Ciudad Universitaria, 5000, Córdoba, Argentina. Tel.: 54-3514681465, ext.: 218. apilatti@unc.edu.ar
} 


\title{
Factors differentiating frequent and sporadic marijuana use in college students
}

\begin{abstract}
Although marijuana use is highly prevalent among university students in Argentina, little is known about the factors that distinguish frequent marijuana users from sporadic users. For this reason, the present study investigated the possible variations in the contexts of consumption, motives for consumption, perceived norms, and protective behavioral strategies among university students with frequent or sporadic marijuana use, and analyzed the usefulness of this set of variables to distinguish between these two types of users. For this purpose, a sample of 1083 Argentine university students completed an online survey on substance use. In particular, this work was conducted with the subsample that reported using marijuana in the last 30 days ( $\mathrm{n}=158 ; 51.3 \%$ female). The survey asked about marijuana use and associated negative consequences, as well as motives for use, perceived social norms, and protective behavioral strategies. As a result, frequent users had a higher number of negative consequences $(\mathrm{M}=4.92)$ than sporadic users $(\mathrm{M}=1.82 ; \mathrm{p} \leq .05)$, and a distinctive profile was found on a set of variables. At the multivariate level, cheerleading motives $(\mathrm{OR}=1.230 ; \mathrm{p} \leq .05)$, protective behavioral strategies $(\mathrm{OR}=.941 ; \mathrm{p} \leq .05)$, solo use $(\mathrm{OR}=2.024 ; \mathrm{p} \leq .05)$, and college party use $(\mathrm{OR}=2.669 ; \mathrm{p} \leq .05)$ discriminated significantly between the two classes of users. Overall, the findings revealed heterogeneous subpopulations of marijuana users that differ not only in use and its consequences, but also in contexts of use and in a set of relevant variables. These distinct subpopulations require different and specific interventions.

Keywords: risk factors, protective factors, marijuana, students.
\end{abstract}

\section{Introducción}

La marihuana, o cannabis, es la sustancia de curso ilegal más consumida en América del Sur (Organización Panamericana de la Salud [OPs], 2018), incluida Argentina (Secretaría de Políticas Integrales sobre Drogas de la Nación Argentina [SEDRONAR], 2017), y su consumo es más prevalente en edades entre los 18 y los 25-29 años —adultez emergente- (Arnett, 2000) que en cualquier otra etapa del desarrollo (Farmer et al., 2015; Patrick et al., 2016; Schulenberg et al., 2018; SEDRONAR, 2017). De hecho, en un estudio con una muestra no representativa de jóvenes universitarios y no universitarios de Argentina (Pilatti et al., 2019), más del $60 \%$ reportó haber consumido marihuana alguna vez en la vida, y más del $40 \%$ en el último año. Asimismo, los resultados correspondientes a una muestra representativa de argentinos que reportaron consumir marihuana en el último año muestran que la modalidad más frecuente de consumo para todos los grupos etarios fue "algunas veces al año" - con un consumo que puede ser hasta mensual- (SEDRONAR, 2017), y que el grupo de 18 a 24 años fue el que presentó la mayor prevalencia de consumo entre semanal y diario $-43.3 \%$ y $12.1 \%$ en adolescentes de 12 a 17 años-.

Adicional a esto, se ha encontrado que aproximadamente el $60 \%$ de los adultos emergentes de Argentina comienza estudios de educación universitaria (Organización para la Cooperación y el Desarrollo Económico [OCDE], 2014), y que el estrés asociado a la vida universitaria (Garett et al., 2017), combinado con un entorno que promueve o facilita el uso de sustancias (Pearson et al., 2019; Pearson, Bravo et al., 2017; Suerken et al., 2016), hace de los años de universidad una etapa de alto riesgo para el inicio y la escalada del consumo de marihuana y los problemas relacionados con su uso (Miech et al., 2017).

Ahora bien, aunque la prevalencia de consumo - particularmente del consumo diario- parece más baja entre universitarios que entre sus pares que no van a la universidad, en los últimos años las tasas de consumo han aumentado de forma más pronunciada entre quienes asisten a la universidad (National Institute on Drug Abuse, 2018). De hecho, en un estudio reciente, que ilustra cuán prevalente es el consumo de marihuana entre universitarios, se encontró que entre el $54.6 \%$ y el $85 \%$ de una muestra de estudiantes universitarios 
de cinco países - EE. UU., Argentina, Uruguay, España y Países Bajos - reportó consumo de marihuana alguna vez en la vida (Bravo, Pearson et al., 2019).

Por otra parte, se ha reportado que los estudiantes universitarios que consumen marihuana tienen un mayor riesgo de experimentar consecuencias negativas académicas, psicológicas y físicas de diferente severidad (Bravo, Pearson et al., 2019; Buckner et al., 2010; Hall, 2009; Napper et al., 2016; Simons et al., 2012); vulnerabilidad que es mayor entre quienes presentan un patrón de uso de alta intensidad o frecuencia (Fischer et al., 2011; Hall \& Degenhardt, 2009; Manning et al., 2019; Pearson, Bravo et al., 2017; Richter et al., 2017; Volkow et al., 2014).

Ahora bien, los motivos de consumo de marihuana, las normas sociales percibidas y las estrategias conductuales de protección son variables cognitivas y conductuales asociadas al consumo de marihuana de estudiantes universitarios (Pearson, Bravo et al., 2017), pero estas variables son también blancos potenciales de intervenciones destinadas a reducir el consumo de marihuana y sus consecuencias negativas.

Los motivos de consumo pueden ser, por una parte, necesidades internas, donde se encuentran los motivos de animación - como el consumo motivado para aumentar el afecto positivo - y los motivos de afrontamiento - como para manejar el afecto negativo_-; o, por otra, necesidades externas, donde se encuentran los motivos de conformidad - como para integrarse en un grupo de pares - y los motivos sociales - como para facilitar las interacciones socialesque motorizan el consumo de marihuana u otras sustancias (Cooper, 1994; Kuntsche et al., 2006). Una quinta dimensión, creada para propiedades específicas de la marihuana, evalúa los motivos de expansión —es decir, para aumentar la creatividad y ser más abierto a experiencias-.

Teniendo esto en cuenta, diferentes trabajos (Bravo, Prince et al., 2017; Bravo, Sotelo et al., 2019) evidencian la asociación entre los motivos y diferentes indicadores del consumo - como frecuencia de uso y problemas derivados - y destacan su papel como antecedentes proximales del consumo de marihuana que median los efectos de - 0 en ocasiones exhiben efectos sinérgicos con- variables más distales, como de personalidad (Bravo, Prince et al., 2017; Bravo, Sotelo et al., 2019).

Por ejemplo, los resultados de un metaanálisis indican que, en comparación con el efecto de todos los otros motivos, los motivos internos de afrontamiento $(\beta=.20)$ y de mejora $(\beta=.26)$, junto con los motivos de expansión $(\beta=.11)$, se asociaron significativamente con la frecuencia de consumo de marihuana, mientras que los motivos de valencia negativa - afrontamiento de situaciones motivacionales aversivas $(\beta=.36)$ y conformidad $(\beta=.07)-\mathrm{se}$ asociaron significativamente a los problemas derivados del consumo (Bresin \& Mekawi, 2019).

Ahora bien, el modelo o teoría del comportamiento social normativo (véase Cialdini et al., 1991) describe la percepción respecto a cómo piensan y actúan integrantes de diferentes grupos sociales - p. ej., grupo de pares, familiares, estudiante universitario "típico", o los amigos más cercanos - sobre qué tanto son aceptadas y valoradas las conductas de consumo, y la forma en que esta percepción influye sobre el propio comportamiento (Napper et al., 2016). Desde este modelo, un tipo particular de norma social percibida refiere a la internalización del consumo de sustancias como un elemento de la cultura universitaria (Osberg et al., 2010), en la cual se encuentra la creencia de que el consumo de sustancias - como la marihuanaconstituye una parte integral de ser estudiante universitario (Osberg et al., 2010; Pearson, Kholodkov et al., 2017). Al respecto, se ha encontrado que un mayor nivel de esta norma se asocia significativa y positivamente con el consumo de marihuana y con la cantidad de consecuencias negativas asociadas (Pearson, Kholodkov et al., 2017 [entre $r=.24 \mathrm{y}$ $r=.36$ ]; Pearson, Liese et al., 2017 [entre $r=.23 \mathrm{y} r=.28$ ]), y que permite distinguir entre estudiantes universitarios con diferentes patrones de consumo de marihuana (Pearson, Liese et al., 2017).

Por otra parte, para abordar el tema se deben tener en cuenta las estrategias conductuales de protección (ECP), entendidas como conductas realizadas inmediatamente antes, durante y después de consumir una sustancia —como la marihuana-, con la finalidad de eliminar o disminuir el grado de intoxicación o las consecuencias negativas asociadas al consumo (Pearson, 2013; Pedersen et al., 2017). En particular, los estudiantes que emplean ECP - p. ej., que evitan consumir marihuana antes de ir a clases o limitan el número de "pitadas" de marihuana, entre otros comportamientos - parecen presentar menor consumo de marihuana y menor cantidad de consecuencias negativas (Bravo, Prince et al., 2017; Pedersen et al., 2016).

Otro aspecto por considerar es que el consumo de sustancias parece variar según los contextos de consumo 
(Beck et al., 2009; Braitman et al., 2017; Spinella et al., 2019), que generalmente refieren a lugares —el entorno físico - o elementos sociales - elementos que caracterizan a las personas presentes - que definen la ocasión de consumo de sustancias como alcohol o marihuana (LippermanKreda et al., 2018). En general, el consumo de sustancias (Keough et al., 2018) —y de marihuana en particular (Beck et al., 2009; Buckner et al., 2012; Phillips et al., 2018)se caracteriza por ser un consumo social, realizado junto $\mathrm{a}-\mathrm{o}$ en presencia de- otras personas. Ciertamente, el consumo de sustancias en solitario es un comportamiento atípico durante la adultez emergente (Phillips et al., 2018) y su mayor frecuencia parece asociarse a mayor cantidad de consecuencias negativas, incluyendo mayor cantidad de síntomas de dependencia (Spinella et al., 2019).

Los estudios anteriormente señalados muestran la relación entre distintos factores de riesgo y protección e indicadores de consumo de marihuana; no obstante, otros estudios han identificado variables que diferencian a universitarios consumidores de marihuana de sus pares no consumidores (Pearson, Liese et al., 2017). La evidencia indica que los consumidores de marihuana no son una población homogénea, puesto que presentan no solo variaciones en el uso, sino también en variables asociadas (Keith et al., 2015; Manning et al., 2019; Pearson, Bravo et al., 2017); además, como se mencionó, quienes consumen de manera más intensa o frecuente son más vulnerables a presentar problemas por el consumo de marihuana.

Partiendo de este contexto, vemos que el aumento sostenido de su consumo en nuestras comunidades resalta la necesidad de comprender los predictores y las consecuencias del consumo frecuente de marihuana (Keith et al., 2015). En este sentido, el presente trabajo tuvo como fin identificar variables que diferenciaran a universitarios con consumo frecuente de marihuana de aquellos que la consumen con menor frecuencia - consumo esporádico-.

Específicamente, los objetivos de este trabajo fueron: (a) describir los contextos de consumo de marihuana - es decir, dónde y con quién se consume - en universitarios con consumo frecuente y esporádico; (b) examinar posibles diferencias en un conjunto de variables - motivos de consumo, normas percibidas y estrategias conductuales de protección - con potencial a ser foco de intervenciones para limitar el consumo de marihuana entre universitarios con consumo frecuente y esporádico; (c) examinar, a nivel multivariado, el efecto de los motivos de consumo, las normas percibidas y las estrategias conductuales de protección para diferenciar entre ambos tipos de consumidores; $y$ (d) identificar contextos de consumo que permitan diferenciar entre estos dos tipos de consumo.

\section{Método}

\section{Diseño de investigación}

Se empleó un diseño descriptivo y comparativo, en el que se realizó la aplicación de encuestas de tipo transversal (Montero \& León, 2007) a una muestra de estudiantes universitarios argentinos.

\section{Muestra y procedimiento}

El presente estudio hace parte de un proyecto más amplio (ELSA: Estudio Longitudinal Sobre Alcohol) que evalúa longitudinalmente el consumo de alcohol y marihuana en estudiantes universitarios argentinos. Para este trabajo, se utilizaron los datos de la primera recolección de la cohorte 2018 de ELSA; la muestra se formó por muestreo accidental, a partir de la invitación a participar a diferentes facultades de dos universidades públicas de la ciudad de Córdoba, Argentina.

La invitación describía el estudio y solicitaba permiso para acceder a los cursos de materias de primer año. En el aula de dictado de clases, el equipo de investigación - conformado por la investigadora responsable y estudiantes avanzados previamente capacitados de la carrera de psicología - explicó el estudio a los estudiantes y los invitó a participar. Posteriormente, se hizo circular una grilla donde los estudiantes interesados en colaborar dejaron sus datos de contacto - correo electrónico, teléfono, redes sociales digitales-.

En total, 3820 estudiantes proporcionaron información de contacto - correo electrónico, número de teléfono celular, y usuario en redes sociales digitales, como Instagram-, y gracias a ello se les envió un correo electrónico con el enlace a una encuesta en línea - en Qualtrics - . Al ingresar a la encuesta, se explicaban los objetivos del estudio, enfatizando la naturaleza voluntaria de la participación y el manejo de la confidencialidad de los datos. Posteriormente, se indicó a los participantes que, al hacer clic en la opción "siguiente", estaban dando su consentimiento informado 
para participar. La encuesta se programó con características que tenían como fin minimizar la probabilidad de datos incompletos - como avisos electrónicos para cada respuesta faltante- . Los participantes recibieron hasta ocho recordatorios — por correo electrónico, teléfono y redes sociales - para completar la encuesta; y, en general, completarla requería de aproximadamente 45 minutos. Para estimular la participación, se sortearon premios entre quienes completaban la encuesta - como vasos térmicos, auriculares, bonos para comida rápida, bonos para libros, entre otros-

En total, 1083 estudiantes $\left(64 \%\right.$ mujeres; $M_{\text {edad }}=$ $19.73 \pm 3.95)$ completaron la encuesta en línea, pero en este estudio se consideraron solo los casos que reportaron consumir marihuana en el último mes $(n=158 ; 51.3 \%$ mujeres $\left[48.7 \%\right.$ hombres], $\left.M_{\text {edad }}=20.08 \pm 4.18\right)$.

\section{Aspectos éticos}

Se respetaron los lineamientos éticos para la investigación con seres humanos establecidos por la Asociación Psicológica Americana (APA, 2017), la declaración de Helsinki de la Asociación Médica Mundial (2017) y la Ley Nacional Argentina 25.326 de Protección de los Datos Personales (2000), de manera que toda la información brindada por parte de los participantes fue manejada de manera confidencial. Todos los participantes dieron su consentimiento informado para hacer parte del estudio, y se destacó la naturaleza voluntaria de la participación y el manejo confidencial de los datos. Adicionalmente, se tomaron medidas para garantizar el respeto de los derechos humanos, se realizó un control para evitar cualquier riesgo emergente, y se garantizó el buen uso y manejo de la información. Finalmente, es importante mencionar que el protocolo de investigación fue evaluado y aprobado por el comité de ética institucional (\#PE 24).

\section{Instrumentos}

\section{Consumo de marihuana}

Siguiendo estudios previos (Pearson, Kholodkov et al., 2017; Pearson, Liese et al., 2017), se indagó la frecuencia de consumo de marihuana en los últimos 30 días - cantidad de días - y la cantidad de marihuana - gramos - consumida en cada día de una semana típica de consumo durante el último mes. Para esto, se utilizó una imagen que describe los gramos correspondientes a diferentes cantidades de marihuana pura o en cigarrillos armados - denominados "porros" en Argentina- De acuerdo con Keith et al. (2015), los participantes fueron agrupados, en función de sus respuestas, en consumidores esporádicos (CE) si tenían de 1 a 9 días de consumo en el último mes; o en consumidores frecuentes $(\mathrm{CF})$ si tenían 10 o más días de consumo en el último mes.

\section{Consecuencias negativas del consumo de marihuana}

Las consecuencias negativas del consumo se midieron con la versión adaptada al español (Bravo, Pearson et al., 2019) del Brief-Marijuana Consequences Questionnaire (B-MACQ) (Simons et al., 2012). Este cuestionario cuenta con 21 ítems dicotómicos -1 = "sí", $0=$ "no"- que miden la ocurrencia de una variedad de consecuencias - desde menores hasta más severas - del consumo de marihuana en los últimos 30 días. Los participantes respondieron indicando si habían experimentado o no las consecuencias enumeradas en cada ítem. El puntaje total se obtiene a partir de la sumatoria de las respuestas positivas y representa la cantidad de consecuencias negativas experimentadas en ese periodo. Los resultados de la adaptación al español, en un estudio con universitarios de Argentina (Bravo, Pearson et al., 2019), muestran que las puntuaciones presentan propiedades psicométricas adecuadas de validez y confiabilidad. Específicamente, entre los participantes de Argentina, los resultados de un análisis factorial confirmatorio (AFC) corroboraron la estructura de ocho factores $\left(\chi_{(1006)}^{2}=1278.28, p<.001\right.$, CFI $=.970$, TLI $=.968$, RMSEA $=.025)$ y los análisis de consistencia interna arrojaron valores entre .74 y .91 (Bravo, Pearson et al., 2019). En el presente estudio, la consistencia interna de las puntuaciones fue también adecuada $(\alpha=.78)$.

Creencias sobre el rol de la marihuana como parte de la vida universitaria

Se utilizó la versión adaptada al español (Pearson et al., 2019) de la Perceived Importance of Marijuana to the College Experience Scale (PIMCES) de Pearson, Kholodkov et al. (2017). La versión en español consta de 8 ítems que se responden con una escala de cinco puntos -de 1 = "Totalmente en desacuerdo" a 5 = "Totalmente de acuerdo"- Cada frase refleja la internalización del consumo de marihuana como componente integral de la 
vida universitaria - p. ej., "La universidad es una época para experimentar con la marihuana"-. Para obtener un puntaje total de las creencias normativas sobre la marihuana en la vida universitaria, se suman los puntajes obtenidos en cada ítem. Estudios previos, con universitarios de Argentina (Pearson et al., 2019), indican que las puntuaciones de la versión adaptada al español poseen propiedades psicométricas adecuadas de validez y confiabilidad. Puntualmente, esta versión mostró un adecuado ajuste a los datos mediante un $\operatorname{AFC}\left(\chi_{(20)}^{2}=424.28, p<.001, \mathrm{CFI}=0.984\right.$, TLI $=0.977$, RMSEA $=0.077)$ y los análisis de consistencia interna arrojaron valores entre .81 y .88 para los participantes de los diferentes países, incluida Argentina. En el presente estudio, las puntuaciones de la escala mostraron un valor de consistencia interna adecuado $(\alpha=.84)$.

\section{Motivos de consumo de marihuana}

Para medir los motivos de consumo de marihuana en los últimos 30 días, se utilizó la versión adaptada al español (Mezquita et al., 2019) de la Marijuana Motives Measure Short Form (MMm-SF) de Simons et al. (1998). Este instrumento consta de 15 ítems distribuidos en cinco dimensiones que miden diferentes motivos de consumo de marihuana: sociales, afrontamiento, conformidad, mejora y expansión. Cada ítem se puntúa con una escala de cinco opciones —de $1=$ "Nunca/Casi nunca" a $5=$ "Siempre/ Casi siempre"- - Un mayor puntaje en cada dimensión es interpretado como una mayor frecuencia de consumo de marihuana por esos motivos. Los resultados de la adaptación al español confirmaron, mediante un AFC, la estructura de cinco factores adecuada $\left(\chi_{(80)}^{2}=121.30\right.$, $p=.002$, CFI $=0.958$, RMSEA $=0.047), \mathrm{y}$ los resultados de consistencia interna mostraron puntajes entre .79 y .90 para las distintas dimensiones; los valores de consistencia interna obtenidos para una muestra de universitarios de diferentes países, incluida Argentina, arrojaron valores entre .79 y .89 (Mezquita et al., 2019). En el presente estudio, los valores de consistencia fueron adecuados en las cinco dimensiones: sociales $(\alpha=.86)$, afrontamiento $(\alpha=.84)$, conformidad $(\alpha=.87)$, animación $(\alpha=.79)$ y expansión $(\alpha=.88)$.

Estrategias conductuales de protección para marihuana

Se utilizó la versión adaptada al español (Herchenroeder et al., 2020) de la Protective Behavioral Strategies for Marijuana Scale (PBSM) de Pedersen et al. (2017). Este instrumento consta de 17 ítems, medidos con una escala de seis puntos — de 1 = "Nunca" a 6 = "Siempre"—, que interrogan respecto a la frecuencia de uso de una variedad de estrategias de protección inmediatamente antes, durante, e inmediatamente después de consumir marihuana. Los puntajes obtenidos en los ítems se suman para obtener un puntaje total de las estrategias conductuales de protección (ECP) utilizadas. La versión en español ha mostrado propiedades psicométricas adecuadas $(\alpha=.83)$ tanto en estudios previos con estudiantes de Argentina (Herchenroeder et al., 2020) como en el presente trabajo $(\alpha=.88)$. Adicionalmente, se realizó un AFC con los datos correspondientes al presente trabajo para confirmar la estructura propuesta, y los resultados mostraron un ajuste adecuado $\left(\chi_{(116)}^{2}=214.86, p<.001\right.$, CFI $=0.939$, RMSEA $=0.075)$.

\section{Contextos de consumo de marihuana}

A partir de investigaciones previas (Lewis et al., 2011; Lipperman-Kreda et al., 2015; Paves et al., 2012), se desarrolló un cuestionario ad hoc para conocer la frecuencia de consumo de marihuana - de 1 = "Nunca/Casi nunca" a 5 = "Siempre/Casi siempre" - en diferentes contextos físicos o lugares - en la casa, fiesta en la casa propia, casa de amigos sin que sea fiesta, fiestas en casa de amigos, fiesta universitaria, fiesta no universitaria, bar, boliche, evento deportivo, calle/plazas/parque, previa [antes de asistir a un evento donde podrá, o no, continuar el consumo] - y sociales — solo/a, con familiares, desconocidos/as, la pareja, un amigo, un pequeño grupo de amigos, un grupo de más de 10 amigos del mismo sexo, grupo pequeño de amigos de ambos sexos, o un grupo de más de 10 amigos de ambos sexos - Para los análisis descriptivos, las respuestas a estas preguntas fueron dicotomizadas - 0 para "Nunca" y 1 para el resto de opciones de respuesta - para reflejar cualquier consumo de marihuana en cada uno de los contextos.

\section{Análisis de datos}

Se utilizó estadística descriptiva — porcentajes - para describir los contextos de consumo de marihuana - dónde y con quién se consume - en consumo esporádico (CE) y consumo frecuente (CF). Para comparar el consumo de marihuana en cada contexto en función de la frecuencia de consumo - CE vS. CF-, se compararon las proporciones de consumidores en cada contexto, y se aplicó la corrección de Bonferroni para reducir el error Tipo $1(.05 / 21=.0024)$. 
Posteriormente, se analizaron las variaciones en los motivos de consumo, normas percibidas y estrategias conductuales de protección entre universitarios con CF y CE. Previo a estos análisis, se indagó la normalidad — mediante análisis de asimetría y curtosis - y homogeneidad de varianza - mediante la prueba de Levene- de estas variables. En las variables que presentaron normalidad y homogeneidad adecuada — cinco de siete; véase Tabla suplementaria— se emplearon análisis estadísticos paramétricos — prueba $t$ de Student-; y, dado que motivos de conformidad presentó valores de asimetría y curtosis no adecuados, y que motivos de afrontamiento no mostró homogeneidad de varianza, estas dos variables fueron analizadas por medio de la prueba no paramétrica U de Mann-Whitney.

De acuerdo con Cohen (1992), el tamaño del efecto en la prueba $t$ de Student se interpretó de la siguiente manera: pequeño $=0.20-0.49$, mediano $=0.50-0.79, y$ grande $\geq 0.80$. Adicionalmente, se examinaron variaciones entre los grupos CE y CF en frecuencia de consumo de marihuana, gramos consumidos en una semana típica y consecuencias negativas inducidas por dicho consumo. Estas variables no presentaron adecuada asimetría y curtosis, ni homogeneidad de varianza, por lo que fueron analizadas mediante la prueba no paramétrica U de Mann-Whitney. El propósito de estos análisis fue confirmar que la segregación de participantes en CE O CF de marihuana genera grupos con características distintivas.

Finalmente, se realizó una regresión logística con el fin de examinar multivariadamente la utilidad de un conjunto de variables para diferenciar entre universitarios con consumo ocasional o consumo frecuente de marihuana - esta regresión explora cómo un conjunto de variables independientes permite distinguir entre diferentes categorías de una medida dependiente categórica - Específicamente, se ingresaron los motivos de consumo, las normas percibidas y las estrategias conductuales de protección como variables independientes, y el tipo de consumidores ( $\mathrm{CE}$ O CF) como dependiente. Otra regresión logística determinó la utilidad de cada contexto de consumo para diferenciar entre CE y CF. Para esto, se estimaron los odds ratios (OR) y los intervalos de confianza (IC) del $95 \%$. Asimismo, se identificó el porcentaje de casos ubicados de manera adecuada a partir del modelo de regresión obtenido. En todos los análisis inferenciales, el valor crítico establecido para cada prueba fue de $\alpha=.05$. Todos los análisis se realizaron con el software SPSS, versión 23.0.

\section{Resultados}

\section{Estadísticos descriptivos}

La Tabla 1 describe - para la muestra total y en función de la frecuencia de consumo de marihuana en los últimos 30 días - el porcentaje de participantes que reportó consumir marihuana en los diferentes contextos físicos y sociales. Para ambos tipos de consumidores, los contextos caracterizados por la presencia de amigos y amigas, particularmente en presencia de la amiga o amigo más cercano, o de grupos pequeños de amigos y amigas, fueron los más prevalentes. En relación con esto, el consumo en la casa de amigos fue el más prevalente para ambos grupos de consumidores.

Por otra parte, los CF, comparados con sus pares CE, reportaron consumir con mayor frecuencia en los diferentes contextos examinados - tanto sociales como físicos-; sin embargo, estas diferencias fueron más marcadas para el consumo en solitario, con familiares, en eventos deportivos, en lugares para bailar, y en fiestas universitarias. De todos los contextos examinados, solo se observaron diferencias estadísticamente significativas en la ocurrencia de consumo en solitario y, seguramente relacionado con esto, el consumo en la casa de los estudiantes.

\section{Diferencias de grupo}

Como se observa en la Tabla 2 - en la cual se presentan los correspondientes estadísticos, los índices de significación asociados y el tamaño del efecto donde corresponda-, los CF exhibieron, en comparación con los CE, un consumo significativamente mayor de marihuana - en frecuencia y cantidad-, y experimentaron un mayor número de consecuencias negativas.

En particular, los CF presentaron un mayor consumo por motivos sociales, de mejora, expansión y afrontamiento que los CE y exhibieron un mayor nivel de normas percibidas respecto al uso de marihuana como elemento central de la vida universitaria; mientras que los CE exhibieron un uso significativamente mayor de estrategias conductuales de protección que sus pares CF. El tamaño del efecto de las diferencias encontradas fue mediano para las normas percibidas y para los motivos sociales, y grande para el resto de las variables. 
Tabla 1.

Ocurrencia de consumo de marihuana en contextos sociales y contextos fisicos (lugares) en universitarios con o sin consumo frecuente de marihuana

\begin{tabular}{|c|c|c|c|c|c|}
\hline & $N$ total & $\begin{array}{c}\text { Consumidores } \\
\text { frecuentes }\end{array}$ & $\begin{array}{c}\text { Consumidores } \\
\text { esporádicos }\end{array}$ & \multicolumn{2}{|c|}{ Comparación } \\
\hline & $\%$ & $\%$ & $\%$ & Ratio & $p$ \\
\hline \multicolumn{6}{|l|}{ Contextos sociales } \\
\hline Solo/a & $30.7(n=47)$ & $78.3(n=18)$ & $22.3(n=29)$ & 3.51 & .0005 \\
\hline Familia & $11.1(n=17)$ & $34.8(n=8)$ & $6.9(n=9)$ & 5.04 & .1719 \\
\hline Desconocidos/as & $24.2(n=37)$ & $47.8(n=11)$ & $20(n=26)$ & 2.39 & .0949 \\
\hline Pareja & $24.8(n=38)$ & $34.8(n=8)$ & $23.1(n=30)$ & 1.51 & .5046 \\
\hline Amigo/a & $81.7(n=125)$ & $91.3(n=21)$ & $80(n=104)$ & 1.14 & .2223 \\
\hline Grupo pequeño del mismo sexo & $68.6(n=105)$ & $78.3(n=18)$ & $66.9(n=87)$ & 1.17 & .3340 \\
\hline Grupo grande del mismo sexo & $27.5(n=42)$ & $52.2(n=12)$ & $23.1(n=30)$ & 2.26 & .0739 \\
\hline Grupo pequeño de ambos sexos & $51.0(n=78)$ & $65.2(n=15)$ & $48.5(n=63)$ & 1.34 & .2484 \\
\hline Grupo grande de ambos sexos & $26.8(n=41)$ & $52.2(n=12)$ & $22.3(n=29)$ & 2.34 & .0672 \\
\hline \multicolumn{6}{|l|}{ Contextos físicos/lugares } \\
\hline Casa & $50.3(n=77)$ & $87(n=20)$ & $43.8(n=57)$ & 1.98 & .0013 \\
\hline Fiesta en casa & $36.6(n=56)$ & $65.2(n=15)$ & $31.5(n=41)$ & 2.07 & .2569 \\
\hline Casa de amigos/as & $78.4(n=120)$ & $78.3(n=18)$ & $78.5(n=102)$ & 0.99 & .9848 \\
\hline Fiesta en casa de amigos/as & $54.9(n=84)$ & $65.2(n=15)$ & $53.1(n=69)$ & 1.22 & .3955 \\
\hline Fiesta universitaria & $15.0(n=23)$ & $39.1(n=9)$ & $10.8(n=14)$ & 3.62 & .1240 \\
\hline Fiesta no universitaria & $44.4(n=68)$ & $65.2(n=15)$ & $40.8(n=53)$ & 1.59 & .0990 \\
\hline Bar & $13.7(n=21)$ & $30.4(n=7)$ & $10.8(n=14)$ & 2.81 & .2773 \\
\hline Boliche & $25.5(n=39)$ & $65.2(n=15)$ & $18.5(n=24)$ & 3.52 & .0055 \\
\hline Recital & $26.1(n=40)$ & $52.2(n=12)$ & $21.5(n=28)$ & 2.43 & .0612 \\
\hline Evento deportivo & $3.9(n=6)$ & $13(n=3)$ & $2.3(n=3)$ & 5.65 & .6478 \\
\hline Calle/plazas/parque & $51.0(n=78)$ & $78.3(n=18)$ & $46.2(n=60)$ & 1.69 & .0191 \\
\hline Previa & $55.6(n=85)$ & $82.6(n=19)$ & $50.8(n=66)$ & 1.63 & .0154 \\
\hline
\end{tabular}

Nota. En negrilla se presentan las prevalencias significativamente más elevadas ( $p \leq .0024$, corrección de Bonferroni [.05/21]) que las prevalencias del grupo de comparación.

Tabla 2.

Medias y desviaciones típicas en motivos de consumo, normas percibidas y estrategias conductuales de protección entre universitarios con o sin consumo frecuente de marihuana

\begin{tabular}{|c|c|c|c|c|c|c|}
\hline & \multicolumn{2}{|c|}{$\begin{array}{l}\text { Consumidores } \\
\text { esporádicos }\end{array}$} & \multicolumn{2}{|c|}{ Consumidores frecuentes } & \multirow[t]{2}{*}{ Prueba $t / U$} & \multirow[t]{2}{*}{$\begin{array}{c}\text { Tamaño de } \\
\text { efecto }\end{array}$} \\
\hline & $M$ & $D T$ & $M$ & $D T$ & & \\
\hline \multicolumn{7}{|l|}{ Indicadores de consumo } \\
\hline Frecuencia & 2.67 & 1.96 & 17.92 & 6.90 & 8.05 & \\
\hline Gramos ST & 1.39 & 1.70 & 6.24 & 6.42 & 3.27 & \\
\hline Consecuencias negativas & 1.83 & 2.15 & 4.92 & 3.96 & 3.96 & \\
\hline \multicolumn{7}{|l|}{ Variables relacionadas } \\
\hline Normas Percibidas & 16.05 & 5.19 & 20.0 & 5.17 & 3.55 & 0.76 \\
\hline ECP & 74.58 & 18.47 & 57.43 & 13.96 & 4.24 & 1.05 \\
\hline \multicolumn{7}{|l|}{ Motivos de consumo } \\
\hline Sociales & 5.45 & 3.07 & 7.78 & 3.54 & 3.29 & 0.70 \\
\hline Afrontamiento & 4.82 & 2.62 & 7.57 & 3.78 & 3.84 & \\
\hline Conformidad & 3.26 & 1.05 & 3.09 & 0.29 & 0.25 & \\
\hline Animación & 8.52 & 3.58 & 11.74 & 3.18 & 4.04 & 0.95 \\
\hline Expansión & 5.87 & 3.37 & 9.17 & 3.88 & 4.23 & 0.91 \\
\hline
\end{tabular}

Nota . ST = semana típica, ECP = estrategias conductuales de protección. En negrilla se presentan las diferencias estadísticamente significativas a $p \leq .05$. En el caso de las pruebas U de Mann-Whitney, se reporta el estadístico Z arrojado por la prueba. 


\section{Regresión logística}

La regresión logística para examinar la utilidad de los motivos de consumo, las normas percibidas y las estrategias conductuales de protección para diferenciar entre los dos grupos de consumidores mostró un adecuado ajuste del modelo (bondad de ajuste de Hosmer-Lemeshow $\left.X^{2}=3.401, p=.907\right)$ y explicó el $45 \%$ de varianza $\left(\mathrm{R}^{2}\right.$ de Nagelkerke $=.449)$. El modelo exhibió un porcentaje global de clasificación correcta, del $86.9 \%$, siendo más eficiente para clasificar adecuadamente a los CE (96.2\%) que a los CF (34.8 \%). De las variables incluidas en el modelo, los motivos de mejora (OR $=1.230$ [IC 95\%: 1.008, 1.501]) y las estrategias conductuales de protección $(\mathrm{OR}=.941$ [IC $95 \%: .909, .973]$ ]) exhibieron odds ratios significativos, lo cual implica que un mayor consumo por motivos de mejora y un menor uso de estrategias conductuales de protección aumentan la probabilidad de pertenecer a la clase de consumo frecuente. En la Tabla 3 se presentan los odds ratios (OR) y los intervalos de confianza (95\%) para las variables incluidas.

Tabla 3.

Regresión logística: estimación de odds ratios

\begin{tabular}{lccc}
\hline & \multicolumn{3}{c}{ CE Vs. CF } \\
\cline { 2 - 4 } & \multirow{2}{*}{ OR } & \multicolumn{2}{c}{ IC 95 \% } \\
\cline { 2 - 4 } & & Inferior & Superior \\
\hline Normas percibidas & 1.042 & 0.940 & 1.156 \\
ECP & $\mathbf{0 . 9 4 1}$ & $\mathbf{0 . 9 0 9}$ & $\mathbf{0 . 9 7 3}$ \\
Motivos de consumo & & & \\
$\quad$ Sociales & 0.985 & 0.806 & 1.204 \\
Afrontamiento & 1.150 & 0.959 & 1.378 \\
Conformidad & 0.388 & 0.066 & 2.275 \\
Animación & $\mathbf{1 . 2 3 0}$ & $\mathbf{1 . 0 0 8}$ & $\mathbf{1 . 5 0 1}$ \\
Expansión & 1.167 & 0.960 & 1.418 \\
\hline
\end{tabular}

Nota $. \mathrm{CE}=$ consumidores esporádicos, $\mathrm{CF}=$ consumidores frecuentes, $\mathrm{ECP}=$ estrategias conductuales de protección, $\mathrm{OR}=$ odds ratios, IC = intervalos de confianza. En negrilla se presentan los odds ratios estadísticamente significativos a $p \leq .05$.

La regresión logística para examinar la utilidad de cada uno de los contextos de consumo de marihuana para distinguir entre CE O CF mostró un adecuado ajuste del modelo $\left(X^{2}=14.822, p=.063\right)$. En particular, el modelo explicó el $53 \%$ de varianza $\left(\mathrm{R}^{2}\right.$ de Nagelkerke $\left.=.534\right)$ y permitió la correcta clasificación del $91.5 \%$ de los participantes - $98.5 \%$ de los CE y $52.2 \%$ de los CF fueron clasificados correctamente-.
De todos los contextos incluidos en el modelo, los únicos con odds ratios significativos fueron el consumo en solitario $(\mathrm{OR}=2.024$ [IC $95 \%$ : 1.090, 3.757]) y el consumo en fiestas universitarias ( $\mathrm{OR}=2.669$ [IC $95 \%$ : 1.051, 6.783]), lo cual implica que una mayor frecuencia de consumo en solitario y en fiestas universitarias aumenta - duplica - la probabilidad de pertenecer a la clase de consumo frecuente. En la Tabla 4 se presentan los odds ratios (OR) y los intervalos de confianza (95\%) para las variables incluidas.

Tabla 4.

Regresión logística: estimación de odds ratios

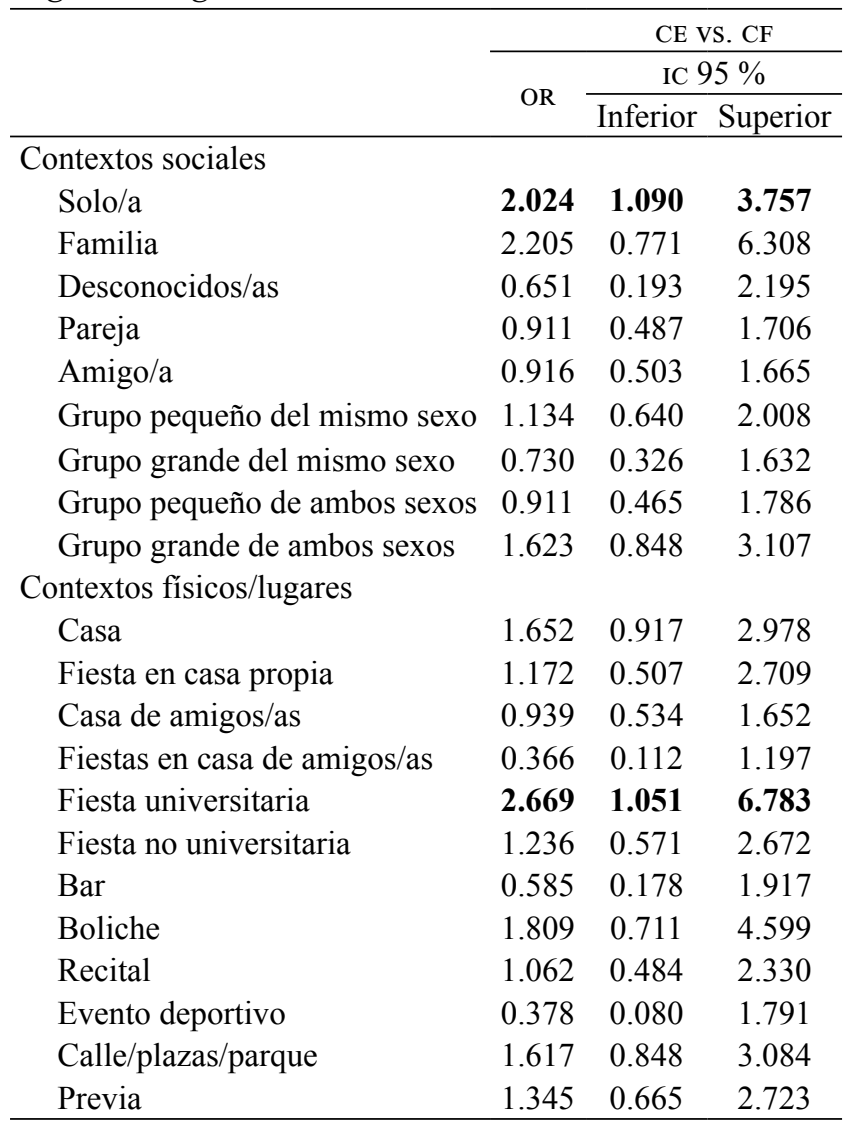

Nota. $\mathrm{CE}=$ consumidores esporádicos, $\mathrm{CF}=$ consumidores frecuentes, $\mathrm{OR}=$ odds ratios, $\mathrm{IC}=$ intervalos de confianza. En negrilla se presentan los odds ratios estadísticamente significativos a $p \leq .05$.

\section{Discusión}

La marihuana es la sustancia ilícita más consumida en Argentina y el mundo. Trabajos recientes indican que, a diferencia de Estados Unidos (Miech et al., 2018) y otros países 
desarrollados en los cuales el consumo de esta sustancia se ha mantenido relativamente estable, en Argentina se han observado incrementos en su uso (Schleimer et al., 2019; SEDRONAR, 2017). A pesar de ello, poco se sabe respecto a las características de los consumidores de marihuana argentinos, y se desconoce en gran parte qué factores discriminan a los consumidores frecuentes de marihuana de aquellos que consumen con menor frecuencia.

Una crítica a la literatura sobre el tema es que usualmente las investigaciones se focalizan en las diferencias entre consumidores y no consumidores de marihuana, pero descuidan la posibilidad de que existan diferencias significativas entre consumidores de marihuana (Pearson, Bravo et al., 2017). En un intento de avanzar sobre esta carencia, el trabajo de Pearson, Bravo et al. (2017) detectó, en una amplia muestra de estudiantes universitarios de Estados Unidos, cuatro clases de consumidores de marihuana; siendo la clase más numerosa la compuesta por consumidores infrecuentes.

El presente trabajo aboga a favor de entender a los consumidores de marihuana argentinos, al menos a aquellos que son estudiantes universitarios, como un grupo heterogéneo. En este sentido, los resultados muestran que los consumidores frecuentes $(\mathrm{CF})$ exhibieron mayor cantidad de consecuencias negativas que los consumidores esporádicos (CE), así como un perfil distintivo en un conjunto de variables. Al analizar estas variables de manera conjunta, aquellas que permitieron diferenciar significativamente entre CF y CE fueron los motivos de animación, las estrategias conductuales de protección (ECP), el consumo en solitario y el consumo en fiestas universitarias. Específicamente, la probabilidad de consumir por motivos de animación fue significativamente mayor en $\mathrm{CF}$ que en $\mathrm{CE}$, y era mucho más probable que los $\mathrm{CF}$ - pero no los $\mathrm{CE}$ - presentaran consumo en solitario y en fiestas universitarias; el uso de ECP fue significativamente menor en CF que en CE.

Estos resultados son parcialmente consistentes con los observados en otros países - p. ej., el trabajo de Pearson, Bravo et al. (2017) observó, de manera similar a nuestro trabajo, que el uso de ECP era menos común en las clases con mayor involucramiento en el consumo de marihuanay son importantes desde un punto de vista aplicado, ya que el uso de ECP puede fomentarse mediante intervenciones estructuradas (Larimer et al., 2007). La evidencia obtenida por nosotros sugiere que los consumidores frecuentes podrían beneficiarse por estos abordajes.
Por otra parte, en sendas muestras de estudiantes universitarios de EE. UU., Pearson, Liese et al. (2017) habían reportado previamente que la percepción del uso de marihuana como una parte integral de la vida universitaria discriminaba entre usuarios y no usuarios de marihuana. No obstante, en nuestro trabajo, la percepción del uso de marihuana como una parte integral de la vida universitaria no permitió discriminar significativamente —en la regresión logística - entre estudiantes universitarios con o sin consumo frecuente de marihuana, pero esto puede deberse a factores intrínsecos de la vida universitaria en Argentina. A diferencia de los norteamericanos, los estudiantes universitarios argentinos no se asocian en agrupaciones - las denominadas "fraternities" o "sororities" - en las cuales las sustancias cumplen un rol predominante, ni viven en complejos habitacionales propios de las universidades, y usualmente eligen universidades de acceso gratuito cercanas a su lugar de residencia. Estos factores pueden explicar por qué no observamos un rol discriminador significativo de la percepción del uso de marihuana como una parte integral de la vida universitaria. Sin embargo, nótese que, a nivel grupal, los estudiantes con CF exhibieron efectivamente un nivel más elevado de normas percibidas que sus pares con CE.

Adicional a esto, encontramos que el consumo de marihuana en fiestas universitarias y en solitario aumentan significativamente la probabilidad de pertenecer a la clase de CF. Aunque el consumo en estos contextos es menos frecuente que en otro tipo de contextos, los resultados sugieren que quienes consumen en estos contextos tienen mayor probabilidad de pertenecer a la clase de CF. En este sentido, sería importante que las autoridades sanitarias y educativas regulen el acceso y disponibilidad de la sustancia en fiestas universitarias. Al respecto, en un estudio realizado con asistentes a fiestas informales de España - aproximadamente la mitad de la muestra eran estudiantes - se indicó que la marihuana era una de las pocas drogas consumidas tanto antes como durante y después de la fiesta; y que era una de las drogas de elección para acompañar el consumo concurrente de otras sustancias - como el alcohol(Fernández-Calderón et al., 2011).

Es interesante mencionar que los contextos más reportados en este trabajo fueron aquellos caracterizados por la presencia de amigos y amigas, particularmente cercanos, $\mathrm{y}$ los grupos de pocos amigos y amigas. En relación con esto, el consumo en casa de amigos fue uno de los contextos de 
mayor frecuencia, mientras que los lugares de menor frecuencia fueron los eventos deportivos, fiestas universitarias y recitales; contextos de consumo de marihuana similares a los que exhibieron mayor prevalencia entre universitarios con consumo de alcohol (Pilatti et al., 2020). Futuros trabajos podrían explorar los contextos de consumo donde ambas sustancias - alcohol y marihuana - se consumen de manera simultánea, ya que este patrón de consumo ejerce efectos diferenciales respecto al consumo individual de cada sustancia (Stevens et al., 2020).

De otro lado, los resultados del presente trabajo, en conjunto con trabajos previos, sugieren que una característica central del consumo de marihuana es que es un consumo con otros. Sin embargo, mientras que el consumo de marihuana ocurre en mayor medida en contextos privados (Phillips et al., 2018), el consumo de alcohol es más frecuente en contextos de fiesta o salida (Lipperman-Kreda et al., 2015; Pilatti et al., 2020). Esto posiblemente se relaciona —al menos parcialmente - con la reglamentación vigente que regula el uso de alcohol y marihuana, que favorece el consumo de alcohol en espacios públicos y el de marihuana en espacios privados.

Por su parte, el resultado de consumo en solitario - predominante de los $\mathrm{CF}$ - es consistente con trabajos que sugieren que aquellos que consumen sustancias en solitario están en más riesgo de escalar a un consumo problemático o de experimentar efectos negativos de dicho consumo, que aquellos que consumen únicamente en contextos caracterizados por la presencia de amigos o familiares (Skrzynski \& Creswell, 2020). El consumo en solitario es un comportamiento atípico durante la adultez emergente (Phillips et al., 2018) y, además, incrementa el riesgo de presentar problemas asociados a estos consumos (Creswell et al., 2014; Keough et al., 2018). De hecho, estudios previos indican que la mayor frecuencia de consumo en solitario de marihuana (Buckner \& Terlecki, 2016) se asocia de manera robusta con indicadores de ansiedad social. Estos resultados, en línea con la clásica teoría de reducción de tensión - que postula que el consumo de sustancias ansiolíticas sirve como estrategia de afrontamiento ante situaciones de emocionalidad negativa (Sinha, 2001) — sugieren que parte de las intervenciones en consumo de sustancias deberían focalizarse en identificar a los consumidores en solitario, especialmente si presentan elevados niveles de ansiedad social $\mathrm{u}$ otro tipo de alteraciones en la regulación emocional. Nótese que los estudiantes universitarios tienen, en comparación con la población general, mayor riesgo de experimentar problemas de salud mental (Bruffaerts et al., 2018), como depresión y ansiedad (Ibrahim et al., 2013), y que quienes consumen marihuana en soledad, comparados con quienes usan la droga en otros contextos sociales, exhiben más frecuencia de uso, más síntomas de dependencia a la marihuana y más motivos de consumo por afrontamiento, según un estudio realizado en Canadá (Spinella et al., 2019).

Por último, es importante mencionar que el consumo de sustancias por motivos de afrontamiento - de estrés u otras situaciones motivacionalmente aversivas - retroalimenta, mediante mecanismos de reforzamiento negativo, el consumo posterior, y puede poner a los individuos en una rápida trayectoria de escalada en el consumo (Merrill $\&$ Thomas, 2013). De hecho, los motivos de consumo por afrontamiento parecen mediar los efectos promotores que factores más distales, como factores de personalidad o psicopatologías subyacentes, ejercen sobre el consumo de sustancias y sus consecuencias (Littlefield et al., 2010). Asimismo, estos motivos están significativamente asociados a la emergencia de problemas inducidos por el consumo de cannabis (Buckner, 2013).

En nuestro trabajo, los estudiantes con CF de marihuana reportaron significativamente más motivos de afrontamiento que sus pares con CE, aunque esta variable no discriminó significativamente entre ambos grupos a nivel multivariado. Para discriminar entre ambos grupos, los motivos de animación fueron mucho más eficientes, lo cual puede deberse a que, si bien ambos motivos se relacionan con los problemas inducidos por el consumo de marihuana, los motivos de afrontamiento lo hacen a partir de un aumento de la frecuencia de consumo en los días de semana, mientras que los motivos de animación se relacionan con los problemas vía un aumento en la frecuencia de consumo tanto en fines de semana como en los días entre semana (Buckner et al., 2019). De esta manera, resulta lógico afirmar que los motivos de animación discriminan mejor a los consumidores de alta frecuencia de aquellos que consumen con menor frecuencia.

Finalmente, los resultados del presente trabajo deben entenderse en el marco de distintas limitaciones, pues, por una parte, el estudio es de tipo transversal, lo cual impide establecer relaciones temporales entre las variables; por otra, 
la muestra fue recogida de manera no probabilística, lo que impide generalizar al conjunto de la población universitaria argentina; y por otra, el tamaño relativamente pequeño de la muestra impide explorar, mediante análisis de clases latentes, la presencia de otras clases de consumidores de marihuana.

Más allá de estos problemas, el estudio identifica contextos que pueden ser particularmente riesgosos para el desarrollo de consumo problemático, y aporta información significativa para identificar subpoblaciones de consumidores de marihuana que requieren de intervenciones o que se favorecerían diferencialmente de recibirlas. En este sentido, de particular preocupación son los subgrupos que exhiben consumo en solitario de marihuana o un consumo motivado para regular el afecto positivo o negativo; y es de destacar que diferentes tipos de intervenciones, como aquellas que intentan aumentar el uso de ECP, han mostrado resultados satisfactorios para reducir el consumo de marihuana. De hecho, diferentes estudios sugieren que la promoción del uso de ECP mediante aplicaciones para celulares (Prince et al., 2020) o vía web (Riggs et al., 2018) reducen de manera significativa el consumo de marihuana, lo cual se sugiere investigar en futuras investigaciones.

\section{Referencias}

Arnett, J. J. (2000). Emerging adulthood. A theory of development from the late teens through the twenties. American Psychology, 55(5), 469-480. https://doi. org/10.1037/0003-066X.55.5.469

Asociación Médica Mundial (2017, abril 22). Declaración de Helsinki - Principios éticos para las investigaciones médicas en seres humanos. https://www.wma.net/ es/policies-post/declaracion-de-helsinki-de-la-amm-prin cipios-eticos-para-las-investigaciones-medicas-en-se res-humanos

Asociación Psicológica Americana (2017, febrero 12). Ethical Principles of Psychologists and Code of Conduct. https://www.apa.org/ethics/code

Beck, K. H., Caldeira, K. M., Vincent, K. B., O’Grady, K. E., Wish, E. D., \& Arria, A. M. (2009). The social context of cannabis use: relationship to cannabis use disorders and depressive symptoms among college students. Addictive Behaviors, 34(9), 764-768. https://doi.org/10.1016/j. addbeh.2009.05.001

Braitman, A. L., Linden-Carmichael, A. N., \& Henson, J. M. (2017). Protective behavioral strategies as a context-specific mediator: A multilevel examination of within-and between-person associations of daily drinking. Experimental and Clinical Psychopharmacology, 25(3), 141-155. https://doi.org/10.1037/pha0000111

Bravo, A. J., Pearson, M. R., Pilatti, A., Mezquita, L., \& Cross-Cultural Addictions Study Team (2019). Negative marijuana-related consequences among college students in five countries: measurement invariance of the Brief Marijuana Consequences Questionnaire. Addiction, 114(10), 1854-1865. https://doi.org/10.1111/add.14646

Bravo, A. J., Prince, M. A., Pearson, M. R., \& Marijuana Outcomes Study Team (2017). Can I Use Marijuana Safely? An Examination of Distal Antecedents, Marijuana Protective Behavioral Strategies, and Marijuana Outcomes. Journal of Studies on Alcohol and Drugs, 78(2), 203-212. https://doi.org/10.15288/ jsad.2017.78.203

Bravo, A. J., Sotelo, M., Pilatti, A., Mezquita, L., Read, J. P., \& Cross-Cultural Addictions Study Team (2019). Depressive symptoms, ruminative thinking, marijuana use motives, and marijuana outcomes: A multiple mediation model among college students in five countries. Drug and Alcohol Dependence, 204, 107558. https://doi. org/10.1016/j.drugalcdep.2019.107558

Bresin, K., \& Mekawi, Y. (2019). Do marijuana use motives matter? Meta-analytic associations with marijuana use frequency and problems. Addictive Behaviors, 99, Article 106102. https://doi.org/10.1016/j.addbeh.2019.106102

Buckner, J. D. (2013). College cannabis use: The unique roles of social norms, motives, and expectancies. Journal of Studies on Alcohol and Drugs, 74(5), 720-726. https:// doi.org/10.15288/jsad.2013.74.720 
Buckner, J. D., Crosby, R. D., Silgado, J., Wonderlich, S. A., \& Schmidt, N. B. (2012). Immediate antecedents of marijuana use: An analysis from ecological momentary assessment. Journal of Behavior Therapy and Experimental Psychiatry, 43(1), 647-655. https://doi. org/10.1016/j.jbtep.2011.09.010

Buckner, J. D., Ecker, A. H., \& Cohen, A. S. (2010). Mental health problems and interest in marijuana treatment among marijuana-using college students. Addictive Behaviors, 35(9), 826-833. https://doi.org/10.1016/j. addbeh.2010.04.001

Buckner, J. D., \& Terlecki, M. A. (2016). Social anxiety and alcohol-related impairment: The mediational impact of solitary drinking. Addictive Behaviors, 58, 7-11. https:// doi.org/10.1016/j.addbeh.2016.02.006

Buckner, J. D., Walukevich, K. A., \& Lewis, E. M. (2019). Cannabis use motives on weekends versus weekdays: Direct and indirect relations with cannabis use and related problems. Addictive Behaviors, 88, 56-60. https:// doi.org/10.1016/j.addbeh.2018.08.012

Bruffaerts, R., Mortier, P., Kiekens, G., Auerbach, R. P., Cuijpers, P., Demyttenaere, K., Green, J. G., Nock, M. K., \& Kessler, R. C. (2018). Mental health problems in college freshmen: Prevalence and academic functioning. Journal of Affective Disorders, 225, 97-103. https://doi. org/10.1016/j.jad.2017.07.044

Cialdini, R. B., Kallgren, C. A., \& Reno, R. R. (1991). A focus theory of normative conduct: A theoretical refinement and reevaluation of the role of norms in human behavior. Advances in Experimental Social Psychology, 24, 201234. https://doi.org/10.1016/S0065-2601(08)60330-5

Cohen, J. (1992). A power primer. Psychological Bulletin, 112(1), 155-159. https://doi.org/10.1037/0033-2909.112. 1.155

Cooper, M. L. (1994). Motivations for alcohol use among adolescents: Development and validation of a four-factor model. Psychological Assessment, 6(2), 117-128. https:// doi.org/10.1037/1040-3590.6.2.117
Creswell, K. G., Chung, T., Clark, D. B., \& Martin, C. S. (2014). Solitary alcohol use in teens is associated with drinking in response to negative affect and predicts alcohol problems in young adulthood. Clinical Psychological Science, 2(5), 602-610. https://doi. org/10.1177/2167702613512795

Farmer, R. F., Seeley, J. R., Kosty, D. B., Gau, J. M., Duncan, S. C., Lynskey, M. T., \& Lewinsohn, P. M. (2015). Internalizing and externalizing psychopathology as predictors of cannabis use disorder onset during adolescence and early adulthood. Psychology of Addictive Behaviors, 29(3), 541-551. https://doi.org/10.1037/adb0000059

Fernandez-Calderón, F., Lozano, O. M., Vidal, C., Ortega, J. G., Vergara, E., González-Sáiz, F., Bilbao, I., Caluente, M., Cano, T., Cid, F., Dominguez, C., Izquierdo, E., \& Pérez, M. I. (2011). Polysubstance use patterns in underground rave attenders: A cluster analysis. Journal of Drug Education, 41(2), 183-202. https://doi.org/10.2190/ DE.41.2.d

Fischer, B., Jeffries, V., Hall, W., Room, R., Goldner, E., \& Rehm, J. (2011). Lower Risk Cannabis use Guidelines for Canada (LRCUG): a narrative review of evidence and recommendations. Canadian Journal of Public Health, 102(5), 324-327. https://doi.org/10.1007/BF03404169

Garett, R., Liu, S., \& Young, S. D. (2017). A longitudinal analysis of stress among incoming college freshmen. Journal of American College Health, 65(5), 331-338. https://doi.org/10.1080/07448481.2017.1312413

Hall, W. (2009). The adverse health effects of cannabis use: what are they, and what are their implications for policy? International Journal of Drug Policy, 20(6), 458-466. https://doi.org/10.1016/j.drugpo.2009.02.013

Hall, W., \& Degenhardt, L. (2009). Adverse health effects of non-medical cannabis use. Lancet (London, England), 374(9698), 1383-1391. https://doi.org/10.1016/S0140-67 36(09)61037-0

Herchenroeder,L.,Mezquita,L.,Bravo,A.J.,Pilatti,A.,Prince, M. A., \& Cross-Cultural Addictions Study Team. (2020). 
A cross-national examination of cannabis protective behavioral strategies' role in the relationship between Big Five personality traits and cannabis outcomes. The American Journal of Drug and Alcohol Abuse, 1-11. https://doi.org/10.1080/00952990.2021.1919689

Ibrahim, A. K., Kelly, S. J., Adams, C. E., \& Glazebrook, C. (2013). A systematic review of studies of depression prevalence in university students. Journal of Psychiatric Research, 47(3), 391-400. https://doi.org/10.1016/j. jpsychires.2012.11.015

Keith, D. R., Hart, C. L., McNeil, M. P., Silver, R., \& Goodwin, R. D. (2015). Frequent marijuana use, binge drinking and mental health problems among undergraduates. The American Journal on Addictions, 24(6), 499506. https://doi.org/10.1111/ajad.12201

Keough, M. T., O'Connor, R. M., \& Stewart, S. H. (2018). Solitary drinking is associated with specific alcohol problems in emerging adults. Addictive Behaviors, 76, 285290. https://doi.org/10.1016/j.addbeh.2017.08.024

Kuntsche, E., Knibbe, R., Gmel, G., \& Engels, R. (2006). Replication and validation of the Drinking Motive Questionnaire Revised (DMQ-R, Cooper, 1994) among adolescentsin Switzerland. EuropeanAddiction Research, 12(3), 161-168. https://doi.org/10.1159/000092118

Larimer, M. E., Lee, C. M., Kilmer, J. R., Fabiano, P. M., Stark, C. B., Geisner, I. M., Mallett, K. A., Lostutter, T. W., Cronce, J. M., Feeney, M., \& Neighbors, C. (2007). Personalized mailed feedback for college drinking prevention: a randomized clinical trial. Journal of Consulting and Clinical Psychology, 75(2), 285-293. https://doi.org/10.1037/0022-006X.75.2.285

Lewis, M. A., Litt, D. M., Blayney, J. A., Lostutter, T. W., Granato, H., Kilmer, J. R., \& Lee, C. M. (2011). They drink how much and where? Normative perceptions by drinking contexts and their association to college students' alcohol consumption. Journal of Studies on Alcohol and Drugs, 72(5), 844-853. https://doi. org/10.15288/jsad.2011.72.844
Ley 25.326 del 30 de octubre de 2000. Ley de Protección de Datos de Carácter Personal, Habeas Data. Argentina. https://www.argentina.gob.ar/justicia/derechofacil/ leysimple/datos-personales

Lipperman-Kreda, S., Mair, C. F., Bersamin, M., Gruenewald, P. J., \& Grube, J. W. (2015). Who drinks where: Youth selection of drinking contexts. Alcoholism: Clinical and Experimental Research, 39(4), 716-723. https://doi.org/10.1111/acer.12670

Lipperman-Kreda, S., Paschall, M. J., Robert F, S., \& Morrison, C. N. (2018). Places and social contexts associated with simultaneous use of alcohol, tobacco and marijuana among young adults. Drug and Alcohol Review, 37(2), 188-195. https://doi.org/10.1111/dar.12537

Littlefield, A. K., Sher, K. J., \& Wood, P. K. (2010). Do changes in drinking motives mediate the relation between personality change and "maturing out" of problem drinking? Journal of Abnormal Psychology, 119(1), 93105. https://doi.org/10.1037/a0017512

Manning, K., Garey, L., Paulus, D. J., Buckner, J. D., Hogan, J. B. D., Schmidt, N. B., \& Zvolensky, M. J. (2019). Typology of cannabis use among adults: A latent class approach to risk and protective factors. Addictive Behaviors, 92, 6-13. https://doi.org/10.1016/j. addbeh.2018.12.008

Merrill, J. E., \& Thomas, S. E. (2013). Interactions between adaptive coping and drinking to cope in predicting naturalistic drinking and drinking following a lab-based psychosocial stressor. Addictive Behaviors, 38(3), 16721678. https://doi.org/10.1016/j.addbeh.2012.10.003

Mezquita, L., Ruiz-Valero, L., Martínez-Gómez, N., Ibáñez, M. I., \& Ortet, G. (2019). Desarrollo y validación de la versión breve del cuestionario de motivos de consumo de marihuana. Adicciones, 31(2), 106-116. https://doi. org/10.20882/adicciones. 979

Miech, R. A., Johnston, L. D., O’Malley, P. M., Bachman, J. G., Schulenberg, J. E., \& Patrick, M. E. (2018). Monitoring the Future National Survey Results on Drug 
Use, 1975-2017: Volume I, Secondary School Students. Institute for Social Research, The University of Michigan. http://monitoringthefuture.org/pubs.html\#monographs

Miech, R. A., Patrick, M. E., O’Malley, P. M., \& Johnston, L. D. (2017). The influence of college attendance on risk for marijuana initiation in the United States: 1977 to 2015. American Journal of Public Health, 107(6), 996-1002. https://doi.org/10.2105/AJPH.2017.303745

Montero, I., \& León, O. G. (2007). A guide for naming research studies in Psychology. International Journal of Clinical and Health Psychology, 7(3), 847-862. http:// www.aepc.es/ijchp/GNEIP07_es.pdf

National Institute on Drug Abuse. (2018, diciembre 14). College-Age and Young Adults. https://www.drugabuse. gov/related-topics/college-age-young-adults

Napper, L. E., Kenney, S. R., Hummer, J. F., Fiorot, S., \& LaBrie, J. W. (2016). Longitudinal relationships among perceived injunctive and descriptive norms and marijuana use. Journal of Studies on Alcohol and Drugs, 77(3), 457-463. https://doi.org/10.15288/jsad.2016.77.457

Organización Panamericana de la Salud [OPS]. (2018). Efectos sociales y para la salud del consumo de cannabis sin fines médicos. Organización Panamericana de la Salud. https://iris.paho.org/handle/10665.2/34944

Organización para la Cooperación y el Desarrollo Económicos [OCDE]. (2014). Education at a Glance 2014: Highlights. OECD Publishing. https://doi. org/10.1787/eag_highlights-2014-en

Osberg, T. M., Atkins, L., Buchholz, L., Shirshova, V., Swiantek, A., Whitley, J., Hartman, S., \& Oquendo, N. (2010). Development and validation of the College Life Alcohol Salience Scale: A measure of beliefs about the role of alcohol in college life. Psychology of Addictive Behaviors, 24(1), 1-12. https://doi.org/10.1037/ a0018197

Patrick, M. E., Schulenberg, J. E., \& O’Malley, P. M. (2016). High school substance use as a predictor of college attendance, completion, and dropout: A national multicohort longitudinal study. Youth \& Society, 48(3), 425-447. https://doi.org/10.1177/0044118X13508961

Paves, A. P., Pedersen, E. R., Hummer, J. F., \& LaBrie, J. W. (2012). Prevalence, social contexts, and risks for prepartying among ethnically diverse college students. Addictive Behaviors, 37(7), 803-810. https://doi.org/10.1016/j. addbeh.2012.03.003

Pearson, M. R. (2013). Use of alcohol protective behavioral strategies among college students: A critical review. Clinical Psychology Review, 33(8), 1025-1040. https:// doi.org/10.1016/j.cpr.2013.08.006

Pearson, M. R., Bravo, A. J., Conner, B. T., \& Marijuana Outcomes Study Team (2017). Distinguishing subpopulations of marijuana users with latent profile analysis. Drug and Alcohol Dependence, 172, 1-8. https://doi. org/10.1016/j.drugalcdep.2016.10.043

Pearson, M. R., Bravo, A. J., Sotelo, M., \& Cross-Cultural Addictions Study Team. (2019). Cross-cultural examination of college marijuana culture in five countries: Measurement invariance of the Perceived Importance of Marijuana to the College Experience Scale. Addictive Behaviors, 96, 11-17. https://doi.org/10.1016/j.addbeh. 2019.04.004

Pearson, M. R., Kholodkov, T., Gray, M. J., \& Marijuana Outcomes Study Team. (2017). Perceived Importance of Marijuana to the College Experience Scale (PIMCES): initial development and validation. Journal of Studies on Alcohol and Drugs, 78(2), 319-324. https://doi. org/10.15288/jsad.2017.78.319

Pearson, M. R., Liese, B. S., Dvorak, R. D., \& Marijuana Outcomes Study Team (2017). College student marijuana involvement: Perceptions, use, and consequences across 11 college campuses. Addictive Behaviors, 66, 8389. https://doi.org/10.1016/j.addbeh.2016.10.019

Pedersen, E. R., Huang, W., Dvorak, R. D., Prince, M. A., \& Hummer, J. F. (2017). The Protective Behavioral Strategies for Marijuana Scale: Further examination 
using item response theory. Psychology of Addictive Behaviors, 31(5), 548-559. https://doi.org/10.1037/ adb0000271

Pedersen, E. R., Hummer, J. F., Rinker, D. V., Traylor, Z. K., \& Neighbors, C. (2016). Measuring protective behavioral strategies for marijuana use among young adults. Journal of Studies on Alcohol and Drugs, 77(3), 441450. https://doi.org/10.15288/jsad.2016.77.441

Phillips, K. T., Phillips, M. M., Lalonde, T. L., \& Prince, M. A. (2018). Does social context matter? An ecological momentary assessment study of marijuana use among college students. Addictive Behaviors, 83, 154-159. https://doi.org/10.1016/j.addbeh.2018.01.004

Pilatti, A., Bravo, A. J., \& Pautassi, R. M. (2020). Contexts of alcohol use: A latent class analysis among Argentinean college students. Drug and Alcohol Dependence, 209, Article 107936. https://doi.org/10.1016/j.drugalcdep .2020 .107936

Pilatti, A., Michelini, Y., Rivarola Montejano, G., Berberian, M., Carrizo, M., \& Pautassi, R. M. (2019). Consumo de alcohol y marihuana en universitarios y no universitarios: relación con factores de vulnerabilidad. Quaderns de Psicologia, 21(2), e1528. https://doi.org/10.5565/rev/ qpsicologia. 1528

Prince, M. A., Collins, R. L., Wilson, S. D., \& Vincent, P. C. (2020). A preliminary test of a brief intervention to lessen young adults' cannabis use: Episode-level smartphone data highlights the role of protective behavioral strategies and exercise. Experimental and Clinical Psychopharmacology, 28(2), 150-156. https://doi. org/10.1037/pha0000301

Richter, L., Pugh, B. S., \& Ball, S. A. (2017). Assessing the risk of marijuana use disorder among adolescents and adults who use marijuana. The American Journal of Drug and Alcohol Abuse, 43(3), 247-260. https://doi.org /10.3109/00952990.2016.1164711

Riggs, N. R., Conner, B. T., Parnes, J. E., Prince, M. A., Shillington, A. M., \& George, M. W. (2018). Marijuana
eCHECKUPTO GO: Effects of a personalized feedback plus protective behavioral strategies intervention for heavy marijuana-using college students. Drug and Alcohol Dependence, 190, 13-19. https://doi.org/10.1016/j. drugalcdep.2018.05.020

Schleimer, J. P., Rivera-Aguirre, A. E., Castillo-Carniglia, A., Laqueur, H. S., Rudolph, K. E., Suárez, H., Ramírez, J., Cadenas, N., Somoza, M., Brasesco, M. V., Martins, S. S., \& Cerdá, M. (2019). Investigating how perceived risk and availability of marijuana relate to marijuana use among adolescents in Argentina, Chile, and Uruguay over time. Drug and Alcohol Dependence, 201, 115-126. https://doi.org/10.1016/j.drugalcdep.2019.03.029

Schulenberg, J. E., Johnston, L. D., O'Malley, P. M., Bachman, J. G., Miech, R. A., \& Patrick, M. E. (2018). Monitoring the Future national survey results on drug use, 1975-2017: Volume 2, College students and adults ages 19-55. Institute for Social Research, The University of Michigan. http://monitoringthefuture.org/pubs.html\# monographs

Secretaría de Políticas Integrales sobre Drogas de la Nación Argentina [SEDRONAR]. (2017). Marihuana: intensidad del consumo. Estudio Nacional en población de 12 a 65 años sobre consumo de sustancias psicoactivas. https://www.argentina.gob.ar/sedronar/investiga cion-y-estadisticas/observatorio-argentino-de-drogas/ estudios/area-de-interes/consumo-de-drogas-en-la -poblacion-general

Simons, J. S., Correia, C. J., Carey, K. B., \& Borsari, B. E. (1998). Validating a five-factor marijuana motives measure: Relations with use, problems, and alcohol motives. Journal of Counseling Psychology, 45(3), 265-273. https://doi.org/10.1037/0022-0167.45.3.265

Simons, J. S., Dvorak, R. D., Merrill, J. E., \& Read, J. P. (2012). Dimensions and severity of marijuana consequences: Development and validation of the Marijuana Consequences Questionnaire (MACQ). Addictive Behaviors, 37(5), 613-621. https://doi.org/10.1016/j. addbeh.2012.01.008 
Sinha, R. (2001). How does stress increase risk of drug abuse and relapse? Psychopharmacology, 158(4), 343-359. https://doi.org/10.1007/s002130100917

Skrzynski, C. J., \& Creswell, K. G. (2020). A systematic review and meta-analysis on the association between solitary drinking and alcohol problems in adults. Addiction, 116(9), 2289-2303. https://doi.org/10.1111/add.15355

Spinella, T. C., Stewart, S. H., \& Barrett, S. P. (2019). Context matters: Characteristics of solitary versus social cannabis use. Drug and Alcohol Review, 38(3), 316-320. https://doi.org/10.1111/dar.12912

Stevens, A. K., Aston, E. R., Gunn, R. L., Sokolovsky, A. W., Treloar Padovano, H., White, H. R., \& Jackson, K. M. (2020). Does the Combination Matter? Examining the Influence of Alcohol and Cannabis Product Combinations on Simultaneous Use and Consequences in Daily Life. Alcoholism: Clinical and Experimental Research, 45(1), 181-193. https://doi.org/10.1111/acer.14494
Suerken, C. K., Reboussin, B. A., Egan, K. L., Sutfin, E. L., Wagoner, K. G., Spangler, J., \& Wolfson, M. (2016). Marijuana use trajectories and academic outcomes among college students. Drug and Alcohol Dependence, 162, 137-145. https://doi.org/10.1016/j. drugalcdep.2016.02.041

Volkow, N. D., Baler, R. D., Compton, W. M., \& Weiss, S. R. (2014). Adverse health effects of marijuana use. New England Journal of Medicine, 370(23), 2219-2227. https://doi.org/10.1056/nejmra1402309 
104

Tabla suplementaria.

Curtosis, asimetría y prueba de homogeneidad de varianzas para las variables motivos de consumo, normas percibidas y estrategias conductuales de protección

\begin{tabular}{|c|c|c|c|c|c|}
\hline & \multicolumn{2}{|c|}{ Consumidores esporádicos } & \multicolumn{2}{|c|}{ Consumidores frecuentes } & \multirow{2}{*}{$\begin{array}{c}p \text { asociada al } \\
\text { estadístico de Levene } \\
p\end{array}$} \\
\hline & Curtosis & Asimetría & Curtosis & Asimetría & \\
\hline \multicolumn{6}{|l|}{ Indicadores de consumo } \\
\hline Frecuencia & 0.152 & 1.085 & -0.971 & 0.427 & .000 \\
\hline Gramos ST & 13.143 & 3.009 & 3.440 & 1.981 & .000 \\
\hline Consecuencias negativas & 1.830 & 1.443 & -0.516 & 0.590 & .000 \\
\hline \multicolumn{6}{|l|}{ Variables relacionadas } \\
\hline Normas percibidas & -0.469 & 0.312 & 0.173 & -0.681 & .694 \\
\hline ECP & 1.268 & -1.021 & 1.712 & -1.081 & .162 \\
\hline \multicolumn{6}{|l|}{ Motivos de consumo } \\
\hline Sociales & 1.430 & 1.417 & -0.873 & 0.129 & .170 \\
\hline Afrontamiento & 2.732 & 1.732 & -0.648 & 0.732 & .005 \\
\hline Conformidad & 57.904 & 6.855 & 8.605 & 3.140 & .128 \\
\hline Animación & -1.006 & 0.209 & 1.691 & -1.300 & .087 \\
\hline Expansión & 0.245 & 1.111 & -1.289 & 0.071 & .181 \\
\hline
\end{tabular}

Nota. ST = semana típica, ECP $=$ estrategias conductuales de protección. 